\title{
Latest trends in fungal epidemiology inform treatment choices and stewardship initiatives
}

János Sinkó*1 \& Jenny Bryan²

'Department of Hematology \& HSCT, St István \& St László Hospital, 5-7 Gyáli u.,1097, Budapest, Hungary Freelance medical journalist, London, UK

*Author for correspondence: +36 $14558100 / 8631$ m janos.sinko@istvankorhaz.hu

\section{2nd European Congress of Clinical Microbiology and Infectious Diseases (ECCMID)}

\section{London, UK, 31 March-3 April 2012}

Twelve months after the WHO launched its global campaign to safeguard current antimicrobial medicines for future generations, antifungal stewardship initiatives were a major focus for the 2012 European Congress of Clinical Microbiology and Infectious Diseases, in London, UK. Speakers from Europe, North and South America and Asia reported significant variations in fungal epidemiology and resistance, and demonstrated the value of multidisciplinary infectious disease advisory teams in monitoring local trends and making recommendations about the most appropriate antifungal treatment.

\section{Trends in fungal epidemiology}

With an expanding patient population at risk of invasive fungal disease (IFD) and growing resistance to antifungal (AF) agents, it is more important than ever to monitor local Aspergillus and Candida species for signs of emerging resistance, Jesús Guinea Ortega (Hospital General Universitario Gregorio Marañón, Spain) advised delegates.

He pointed out that half of cases of invasive candidiasis (IC) are not diagnosed by blood culture, so it is essential to be aware of patients at greatest risk of infection [1]. Guinea Ortega explained that high-risk groups for IC are patients with intravascular catheters, those undergoing intra-abdominal surgery or on broad-spectrum antibiotics, as well as premature infants and the elderly. Invasive aspergillosis (IA) also remains a diagnostic challenge. For IA, hematological cancer patients, solid organ or stem cell recipients and those with chronic obstructive pulmonary disease on long-term corticosteroids are at greatest risk.

Guinea Ortega presented the latest data from a prospective candidemia surveillance program in five areas of Spain with a total population of over 7 million people, which showed an annual incidence of candidemia of 10.7 per 100,000 population and 0.78 per 1000 admissions [2]. Of these, $89 \%$ of cases were in-patients $(40 \%$ intensive care unit [ICU], 32\% medical and $24 \%$ surgical) and $38 \%$ had malignancies. A total of $76 \%$ of patients had a central venous catheter and $51 \%$ had undergone surgery in the
3 months prior to candidemia. Guinea Ortega explained that $46 \%$ of cases were caused by Candida albicans, but unlike centers in some parts of Europe and USA, where Candida glabrata is the most common nonalbicans strain, Candida parapsilosis (25\%) was most frequent in Spain. AF susceptibility was tested in 650 isolates and the overall rate of fluconazole resistance ( $\mathrm{MIC}>4 \mu \mathrm{g} / \mathrm{ml}$ ) was $14.6 \%$. Overall mortality was $38 \%$, with the highest rate in the $2 \%$ of patients with Candida krusei (odds ratio: 3.9). Removal of central venous catheter within the first $48 \mathrm{~h}$ of infection was protective against early mortality (odds ratio: 0.37 ).

Guinea Ortega also highlighted the potential for horizontal transmission of species causing candidemia in hospitals. During a 4-year period, strains of $C$. albicans isolated from blood cultures from 208 patients with candidemia in his hospital showed high genotypic diversity, with up to $25 \%$ of patients infected with a genotype found in at least one other patient [3]. However, in up to $65 \%$ of cases, patients were not located in the same department at diagnosis.

From South America, Maria Luiza Moretti and colleagues (State University of Campinas, Brazil) reported a doubling in incidence of $C$. glabrata in a large tertiary hospital between 2006 and 2010, from 12 to $24 \%$ in 2010 , while the frequency of C. parapsilosis was 20\% in both 2006 and 2010 [4]. The researchers pointed out that their high incidence of C. glabrata was not found in other parts of Brazil, and nearly half of their cases occurred in patients with solid malignancies.

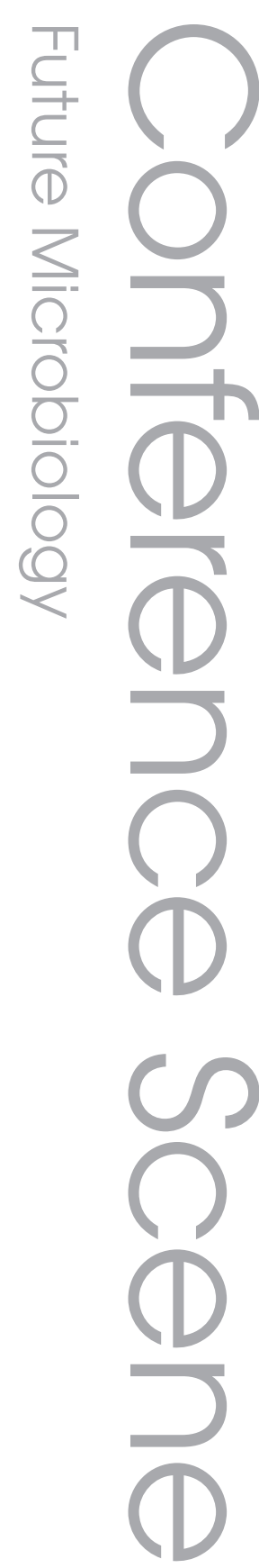

\section{Keywords}

= antifungal = Candida

- invasive aspergillosis

n resistance n stewardship

Future $\because: \%$
Medicine $: .$. 
From Europe, Laurent Tadec (Université de Nantes, France) reported that, contrary to many studies, there had been no emergence of nonalbicans strains during a 7-year period [5]. As in Spain, C. parapsilosis was more common than C. glabrata (15 vs 10\%), although Candida tropicalis was the most common cause of candidemia in the hematology department, which had the highest prevalence of nonalbicans strains in the hospital.

Data from the first nationwide analysis of candidemia frequency in China showed that C. albicans was the predominant species (34\%), followed by C. parapsilosis (18\%), C. tropicalis (15\%) and C. glabrata (11\%) [6] .

The latest data from the Sorveglianza Epidemiologica Infezioni Fungine nelle Emopatie Maligne (SEIFEM) registry underlined the importance of checking for prehospital risk factors for IFD when admitting patients [7]. In addition to well-established risk factors, such as performance status $(\mathrm{p}<0.001)$, chronic obstructive pulmonary disease $(\mathrm{p}=0.04)$, urinary catheter $(\mathrm{p}<0.001)$ and neutropenia (<500 neutrophils/ $\mu l,>7$ days; $\mathrm{p}=0.03)$, nonsignificant trends were seen for environmental risk factors, including a construction site near the home within the last 3 months and home restructuring in the last 6 months. For mold infections only, patients living in a flat had a higher risk than those living in a house with a garden $(p=0.03)$. IC risk factors included chronic kidney failure $(p=0.006)$ and liver diseases $(\mathrm{p}<0.001)$.

A major analysis of fungemia epidemiology and mortality in 145,000 patients taking part in cancer trials at 13 European Organisation for Research and Treatment of Cancer (EORTC) centers in eight countries confirmed the high incidence of nonalbicans candidemia (54\%) in this cohort, although survival was not significantly different from patients with C. albicans [8]. Fungemia occurred in $2.3 \%$ of patients, ranging from $1.5 \%$ in patients with solid tumors to $14.6 \%$ in recipients of hematopoietic stem cell transplantation for hematological malignancy. The 12 -week survival rate was $45 \%$, with fungemia the sole cause of death in $5 \%$ and contributing to mortality in $44 \%$.

\section{Microbiological diagnosis \& species identification}

At ECCMID 2012, there was a sharp contrast between the rapid progress in laboratory methodology and the somewhat sluggish developments in antimicrobial compounds. The matrix-assisted laser desorption/ionization time-of-flight (MALDI-TOF) method seemed to 'come of age' - playing a lead role in the mycological laboratory. John Haigh and coworkers (Barts and The London NHS Trust, UK) tested the usefulness of this mass spectrometry-based method for rapid identification of yeast species in comparison with PCR methods [9]. With MALDI-TOF, 88\% (113 out of 128) of strains could be correctly identified. This result proved a nonsignificant advantage over the $82 \%$ performance of PCR tests. The authors concluded that, where available, MALDI-TOF could be a reliable method for the identification of yeast isolates with a reasonable turnaround time (24-36 h) and low running costs.

\section{Resistance issues}

Direct detection of resistance to AF agents in clinical specimens using molecular tools holds great potential benefit for patients, but clinicians must first address the large number of different resistance mechanisms that have been identified, suggested Corne Klaassen (Canisius Wilhelmina Hospital, The Netherlands). Taking azoles as an example, Klaassen explained that there are four main resistance mechanisms: point mutations in target genes (CYP51A in Aspergillus and ERG11 in Candida); increased expression of target genes; increased expression of efflux pumps; and species-specific intrinsic resistance.

In a recent study, a novel mixed-format realtime PCR assay was used to identify wild-type CYP51A and point mutations conferring azole resistance in Aspergillus fumigatus isolates [10]. Klaassen described how hybridization of ssDNA detection probes with single-strand target gene DNA enabled the researchers to differentiate between susceptible and resistant strains, using fluorescent dyes. Four triazole-resistant isolates were identified, all of which contained an identical combination of mutations leading to multitriazole resistance. Molecular testing results were totally concordant with phenotypic susceptibility testing.

Given this success, and the heterogeneity of microbial resistance mechanisms, Klaassen recommended focusing on species that are well studied, such as A. fumigatus and Candida, and using blood rather than other fluid samples. To emphasize this advice, he described a fatal case of IA in which bronchoalveolar lavage samples failed to show a resistant strain (TR/L98H) until week 5 of tests, and then a confusing pattern of sensitive and resistant strains. By contrast, the TR/L98H isolate was visible in serum by week 3, and Klaassen concluded that an organism that 
is detectable in serum is usually the most likely source of relevant infection.

Azole resistance was a recurring theme at the congress, with data presented from The Netherlands and the UK, where environmental factors are thought to be playing a role. Guinea Ortega cited data from a recent study of over 2000 isolates tested in 1385 patients in The Netherlands, which showed a prevalence of itraconazole resistance in A. fumigatus of $5.3 \%$, with levels ranging from 0.8 to $9.5 \%$ depending on which part of the country the isolates came from [11]. Patients with a hematologic or oncologic disease were at greatest risk of itraconazole-resistant infection, and $64 \%$ of patients with resistant isolates were azole naive. Mortality in patients with resistant isolates was $88 \%$.

In a study of AF susceptibility in Candida species in the UK, Abid Hussain (Heart of England Foundation Trust, UK) reported that $44 \%$ of 39 blood culture isolates were positive for $C$. albicans, as were $45 \%$ of 62 isolates from nonsterile sites [12]. He also demonstrated the impact of the evolution and harmonization of Clinical and Laboratory Standards Institute (CLSI) and European Committee on Antimicrobial Susceptibility Testing (EUCAST) breakpoints in clinical practice, with fluconazole resistance in nonalbicans isolates from blood cultures rising from $42 \%$ using old MIC breakpoints to $78 \%$ with the new breakpoints. Against this background, Hussain recommended that patients with proven or probable candidemia should be managed empirically with an echinocandin and de-escalated to fluconazole in the event of proven susceptibility.

Animal studies supporting previously reported in vitro effects of ibuprofen in reversing fluconazole resistance due to efflux pump overexpression were presented by Sofia Costa-de-Oliveira and colleagues (Porto University, Portugal) [13]. Mice were infected with fluconazole-sensitive and -resistant strains of Candida, and received intraperitoneal fluconazole, ibuprofen or combined treatment with fluconazole and ibuprofen $3 \mathrm{~h}$ after microbial challenge and once a day thereafter for 4 days. Ibuprofen decreased azole MIC values, and the resistant phenotype changed to sensitive. Microarray analysis showed overexpression of CDR11, ERG251, CDR4 and the transcription factor UPC2 in the resistant strain, but these genes were downregulated in the ibuprofen-treated and sensitive strains. Fluconazole was found to be effective only in the treatment of infection by the sensitive Candida strain, but animals infected with the resistant strain and treated with fluconazole and ibuprofen also showed a significant decrease in fungal burden. Ibuprofen alone showed no AF activity. The researchers concluded that in vivo synergy between fluconazole and ibuprofen offers hope for better management of AF resistance conferred by efflux pump overexpression.

\section{Update on AF treatment}

To support decision-making for AF prophylaxis in allogeneic hematopoietic cell transplantation patients, a systematic review of major prophylaxis trials was carried out and the results presented at ECCMID [14].

The review, authored jointly by leading investigators involved in the five trials [15-19], concluded that voriconazole and posaconazole are better choices for prophylaxis in this patient group than itraconazole. Comparing proven/ probable IA rates at day 180 from the studies, the review showed that voriconazole prophylaxis had an $85 \%$ probability of a favorable outcome relative to fluconazole, compared with $80 \%$ for posaconazole and $69 \%$ for itraconazole. Posaconazole had a $45 \%$ probability of achieving the lowest IA rate of the four agents, compared with $42 \%$ for voriconazole and $10 \%$ for itraconazole. The group concluded that, in the absence of data on the comparative efficacy of voriconazole and posaconazole, ease of use, fungal species susceptibility and cost are likely to determine the final prescribing decision.

Oliver Cornely and colleagues (University of Cologne, Germany) presented evidence from a comparison of the cost of prophylaxis with posaconazole and topical polyene in patients with acute myelogenous leukemia and myelodysplastic syndrome [20]. Hospital costs for patients treated before and after the introduction of posaconazole prophylaxis were compared, and it was shown that the average costs per patient were $€ 21,040$ in the posaconazole group and $€ 23,169$ in the topical polyene group. AF treatment costs were slightly higher for posaconazole (€4580 vs $€ 4019$ ), but these were more than offset by lower costs of antibacterial and antiviral agents and diagnostic procedures, and shorter times in the ICU (1.79 days per patient for the posaconazole group compared with 3.83 days per patient in the topical polyene group). This resulted in a trend towards cost-saving from use of posaconazole prophylaxis in patients receiving remission-induction chemotherapy.

The same researchers also compared the costs of treating candidemia in ICU patients, using newer agents (echinocandins, liposomal 
amphotericin B or voriconazole) or conventional AFs (amphotericin B deoxycholate or fluconazole) [21]. This analysis showed that the condition of patients treated with the newer agents tended to be worse than those treated with older agents, and patients were more likely to have nonalbicans Candida infection. The direct and indirect costs of treatment with newer agents were higher than with older agents (€41,060 vs $€ 28,885$ per patient and $€ 1202$ vs $€ 1087$, respectively). However, as the researchers pointed out, post-hospital and 1-year survival rates were similar for the two groups (56 vs $50 \%$ and 44 vs $33 \%$, respectively), showing that comparable outcomes could be achieved with sicker patients using newer AFs, as were seen with less ill patients treated with conventional drugs.

William Hope (University of Manchester, UK) discussed initial findings with a new approach to optimizing azole dose adjustment to predefined serum concentration targets [22]. He used recently published pharmacokinetic data from 64 healthy volunteers and patients with proven/probable IA treated with voriconazole [23] to develop a program to predict voriconazole dosages required to achieve a predefined serum trough concentration of $1 \mathrm{mg} / \mathrm{l}$. Hope reported that the concentration-time profile of each of ten hematological stem cell transplantation patients treated with a standard voriconazole regimen was predicted with a high degree of accuracy, despite some patients having nonlinear pharmacokinetics. Hope explained that the process of dosage optimization could begin with the first dosing interval, meaning that therapeutic and nontoxic serum concentrations can potentially be achieved in the first 48-72 h of dosing.

Paolo Campoli and colleagues (McGill University, QC, Canada) presented research aimed at improving the understanding of azole kinetics at the subcellular level. After previous research had suggested that intracellular levels of posaconazole may be more relevant to therapeutic efficacy than serum levels [24], Campoli et al. observed that posaconazole was predominantly found in host cell membranes in concentrations sufficient to inhibit growth and prevent fungal damage [25]. Subsequent research, presented at ECCMID, showed that during infection, posaconazole transferred from host cell membranes to fungal membranes and was concentrated within the endoplasmic reticulum (ER) [26]. The researchers pointed out that, as the target for posaconazole, CYP51A, is found within the ER, the drug's localization may be relevant to its AF activity. As the addition of voriconazole did not result in competitive inhibition of posaconazole within the ER membrane, it was suggested that ER concentration is specific to posaconazole. Further investigation will be needed to prove the clinical relevance of these data.

For patients with some of the most difficult to treat fungal infections, caused by Scedosporium or Pseudallescheria species, in vitro susceptibility data suggest a potential therapeutic role for voriconazole, posaconazole and micafungin in Pseudallescheria apiosperma, Pseudallescheria boydii and Scedosporium prolificans infections [27], while for those with another therapeutic challenge $-C$. parapsilosis catheter infections - there was evidence from an animal model of the efficacy of anidulafungin, using the AF lock technique [28].

Promising data were also available concerning the use of combination treatment as primary therapy for IA - an approach that has previously been generally reserved for salvage therapy [29] . Kieren Marr (Johns Hopkins Hospital, MD, USA) and colleagues reported results showing a trend towards reduced all cause mortality at 6 weeks with the combination of voriconazole and anidulafungin compared with voriconazole monotherapy (26 out of 135 [19.3\%] vs 39 out of 142 [27.5\%], respectively). Although this result was not statistically significant, a post hoc analysis of 218 patients with probable IA based on detection of galactomannan in bronchoalveolar lavage or serum did show significance (15.7 vs $27.3 \% ; \mathrm{p}<0.05)$.

\section{Impact of AF stewardship initiatives}

Multidisciplinary AF stewardship initiatives aimed at improving clinical outcomes, avoiding adverse drug-related events, reducing emergence of resistance and controlling costs were reported at this year's congress.

At the University of Cologne, the introduction of an infectious disease consulting service, run jointly by clinical microbiology and infectious diseases departments has been well received by clinicians. Cornely reported a rapid increase in electronic requests for advice, with the service now handling approximately 1400 requests per year - equivalent to one per bed of the hospital. Approximately half of requests are made by surgical disciplines, and Cornely explained that the most typical advice given to clinicians is to stop anti-infectives, adjust the dose, focus treatment or make a proper diagnosis for the infection being treated. 


\section{Developments in newer AFs}

Although limited progress in the development of new AFs was reported at ECCMID this year, Dimitrios Kontoyiannis (MD Anderson Cancer Center, TX, USA) highlighted the novel oral glucan synthase inhibitor, MK-3118, as one of the more promising agents. Active against both Candida and Aspergillus, MK-3118 has demonstrated in vitro inhibition of glucan synthesis comparable to that of caspofungin. In a Phase I multidosing study, MK-3118 was found to be well tolerated in doses up to $800 \mathrm{mg}$ for up to 28 days, with a favorable pharmacokinetic profile.

Kontoyiannis reported that Phase II and III studies are underway comparing a novel oral formulation of posaconazole with intravenous treatment in patients at high risk of IFD. This follows healthy volunteer studies showing good systemic exposure with improved oral posaconazole, with or without food, and dose-finding studies favoring a 300-mg daily dose. Kontoyiannis also reviewed studies of the new azole, isavuconazole, showing high bioavailability for the oral capsule, with no effect of food or acid suppression, and once-daily dosing. He added that isavuconazole is active against Candida and Aspergillus infections, with modest activity against Rhizopus and Scedosporium. Kontonyiannis said that little progress had been made with immunoadjuvant therapies for fungal infection, and that a combination of lack of research skills to work on natural products, limited delivery vehicles and ongoing regulatory issues was impeding developments. He concluded that an orchestrated strategy is needed to develop good biomarkers for measuring the efficacy of novel vaccines and antibody treatments in order to achieve more rapid progress.

\section{Financial \& competing interests disclosure \\ $J$ Bryan's ECCMID attendance and her contribution to the writing of the report was supported by MSD. The authors have no other relevant affiliations or financial involvement with any organization or entity with a financial interest in or financial conflict with the sub- ject matter or materials discussed in the manuscript apart from those disclosed. \\ No writing assistance was utilized in the production of this manuscript.}

\section{References}

1. Guinea Ortega JV. Epidemiology of invasive fungal infections: an update. Clin. Microbiol. Infect. 18(Suppl. S3), 30 (S217) (2012).

2. Puig M, Garnacho J, Padilla B et al. Epidemiology, risk factors for mortality and fluconazole susceptibility in a populationbased surveillance of candidaemia in Spain. Clin. Microbiol. Infect. 18(Suppl. S3), 9 (O110) (2012).

3. Escribano P, Recio S, Peláez T et al. Clonal diversity of Candida albicans isolates causing candidaemia over a 4-year period: patients located in different departments can be infected by identical genotypes. Presented at: 22nd European Congress of Clinical Microbiology and Infectious Diseases (ECCMID). London, UK, 31 March-3 April 2012 (Poster 745).

4. Moretti ML, Trabasso P, Lyra L et al. Is the incidence of candidaemia caused by Candida glabrata increasing in Brazil? Five-year surveillance of Candida bloodstream infection in a university reference hospital in southeastern Brazil. Presented at: 22nd European Congress of Clinical Microbiology and Infectious Diseases (ECCMID). London, UK, 31 March-3 April 2012 (Poster 783).

5. Tadec L, Talarmin J-P, Le Pape P et al. Epidemiological trends of nosocomial candidaemia over a 7-year period at Nantes University Hospital, France. Presented at: 22nd European Congress of Clinical Microbiology and Infectious Diseases (ECCMID). London, UK, 31 March-3 April 2012 (Poster 781).

6. Wang H, Xiao M, Xu Y. Results from the China Hospital Invasive Fungal Surveillance Net 2009-2010: an analysis of susceptibilities of yeast species to fluconazole and voriconazole as determined by CLSI standardised disc diffusion. Clin. Microbiol. Infect. 18(Suppl. S3), 10 (O113) (2012).

7. Caira M, Candoni A, Busca A et al. Pre-hospital risk factors for invasive fungal disease in patients with acute myeloid leukemia at diagnosis: preliminary results from the SEIFEM 2010-study. Presented at: 22nd European Congress of Clinical Microbiology and Infectious Diseases (ECCMID). London, UK, 31 March-3 April 2012 (Poster 811).

8. Cornely OA, Gachot B, Akan H et al. Epidemiology and mortality of fungaemia in cancer patients - a clinical cohort of the Infectious Diseases Group (IDG) of the European Organization for Research and Treatment of Cancer (EORTC 65031). Clin. Microbiol. Infect. 18(Suppl. S3), 9 (O109) (2012).

9. Haigh JD, Petrou M, Wilks M. Rapid identification of yeast species comparing MALDITOF and Prove-it ${ }^{\mathrm{TM}}$ PCR methods. Presented at: 22nd European Congress of Clinical Microbiology and Infectious Diseases
(ECCMID). London, UK, 31 March-3 April 2012 (Poster 850).

10. Klaassen CH, de Valk HA, Curfs-Breuker IM, Meis JF. Novel mixed-format real-time PCR assay to detect mutations conferring resistance to triazoles in Aspergillus fumigatus and prevalence of multi-triazole resistance among clinical isolates in The Netherlands. J. Antimicrob. Chemother. 65(5), 901-905 (2010).

11. van der Linden JW, Snelders E, Kampinga GA et al. Clinical implications of azole resistance in Aspergillus fumigatus, The Netherlands, 2007-2009. Emerg. Infect. Dis. 17(10), 1846-1854 (2011).

12. Hussain A. Species distribution and anti-fungal susceptibility of Candida spp at the Heart of England Foundation Trust, United Kingdom. Presented at: 22nd European Congress of Clinical Microbiology and Infectious Diseases (ECCMID). London, UK, 31 March-3 April 2012 (Poster 749).

13. Costa-de-Oleiveria S, Miranda IM, Ricardo E et al. Effective reversion of fluconazole resistance by ibuprofen in an animal model. Clin. Microbiol. Infect. 18(Suppl. S3), 11 (O118) (2012).

14. Bow EJ, Vanness DJ, Cordonnier C et al. Mixed treatment comparison of randomised clinical trials of primary antifungal prophylaxis in allogeneic haematopoietic cell transplant recipients - focus on invasive aspergillosis. Presented at: 22nd European 
Congress of Clinical Microbiology and Infectious Diseases (ECCMID). London, UK, 31 March-3 April 2012 (Poster 867).

15. Winston DJ, Maziarz RT, Chandrasekar PH et al. Intravenous and oral itraconazole versus intravenous and oral fluconazole for long-term antifungal prophylaxis in allogeneic hematopoietic stem-cell transplant recipients. A multicenter, randomized trial. Ann. Intern. Med. 138(9), 705-713 (2003).

16. Marr KA, Crippa F, Leisenring W et al. Itraconazole versus fluconazole for prevention of fungal infections in patients receiving allogeneic stem cell transplants. Blood 103(4), 1527-1533 (2004).

17. Ullmann AJ, Lipton JH, Vesole DH et al. Posaconazole or fluconazole for prophylaxis in severe graft-versus-host disease. N. Engl. J. Med. 356(4), 335-347 (2007).

18. Wingard JR, Carter SL, Walsh TJ et al. Randomized, double-blind trial of fluconazole versus voriconazole for prevention of invasive fungal infection after allogeneic hematopoietic cell transplantation. Blood 116(24), 5111-5118 (2010).

19. Marks DI, Pagliuca A, Kibbler CC et al. Voriconazole versus itraconazole for antifungal prophylaxis following allogeneic haematopoietic stem-cell transplantation. Br. J. Haematol. 155(3), 318-327 (2011).

20. Heimann $S$, Cornely OA, Wisplinghoff $\mathrm{H}$ et al. Development of treatment costs of patients undergoing remission induction chemotherapy: a historical comparison before and after introduction of posaconazole prophylaxis. Presented at: 22nd European Congress of Clinical Microbiology and Infectious Diseases (ECCMID). London, UK, 31 March-3 April 2012 (Poster 853).

21. Heimann S, Cornely OA, Wisplinghoff $\mathrm{H}$ et al. Cost-of-illness analysis of candidemia in patients on the intensive care unit. Presented at: 22nd European Congress of Clinical Microbiology and Infectious Diseases (ECCMID). London, UK, 31 March-3 April 2012 (Poster 854).

22. Hope W, VanGuilder M, Donnelly P et al. Software to enable optimally precise dosage adjustment of voriconazole to achieve predefined serum concentration targets in critically patients. Clin. Microbiol. Infect. 18(Suppl. S3), 44 (O299) (2012).

23. Hope WW. Population pharmacokinetics of voriconazole in adults. Antimicrob. Agents Chemother. 56(1), 526-531 (2012).

24. Farowski F, Cornely OA, Vehreschild JJ et al. Intracellular concentrations of posaconazole in different compartments of peripheral blood. Antimicrob. Agents Chemother. 54(7), 2928-2931 (2010).

25. Campoli P, Al Abdallah Q, Robitaille R et al. Concentration of antifungal agents within host cell membranes: a new paradigm governing the efficacy of prophylaxis. Antimicrob. Agents Chemother. 55(12), 5732-5739 (2011).

26. Campoli P, Attias O, Kristof AS et al. Posaconazole concentrates within the endoplasmic reticulum of host and fungal cells. Presented at: 22nd European Congress of Clinical Microbiology and Infectious Diseases (ECCMID). London, UK, 31 March-3 April 2012 (Poster 830).

27. Lackner M, de Hoog S, Verweij P et al. Antifungal susceptibility patterns of Scedosporium and Pseudallescheria species. Clin. Microbiol. Infect. 18(Suppl. S3), 10 (O112) (2012).

28. Gavalda K, Orbegozo CM, Gomis X et al. Efficacy of anidulafungin in the treatment of experimental Candida parapsilosis catheter infection using the antifungal-lock technique. Clin. Microbiol. Infect. 18(Suppl. S3), 11 (O116) (2012).

29. Marr KA, Schlamm H, Rottinghau ST et al. A randomised, double-blind study of combination antifungal therapy with voriconazole and anidulafungin versus voriconazole monotherapy for primary treatment invasive aspergillosis. Presented at: 22nd European Congress of Clinical Microbiology and Infectious Diseases (ECCMID). London, UK, 31 March-3 April 2012 (Poster LB2812). 